\title{
Linear Modeling of Possible Mechanisms for Parkinson Tremor Generation
}

\author{
Peter LöhNBerg \\ Bio-information Group, Department of Electrical Engineering, \\ Twente University of Technology, Enschede, The Netherlands
}

Received July 24, 1978

\begin{abstract}
The power of Parkinson tremor is expressed in terms of possibly changed frequency response functions between relevant variables in the neuromuscular system. The derivation starts out from a linear loopless equivalent model of mechanisms for general tremor generation. Hypothetical changes in this model from the substrate of the disease are indicated, and possible ones are inferred from literature about experiments on patients. The result indicates that in these patients tremor appears to have been generated in loops, which did not include the brain area which in surgery usually is inactivated. For some patients in the literature, these loops could involve muscle length receptors, the static sensitivity of which may have been enlarged by pathological brain activity.
\end{abstract}

\section{INTRODUCTION}

A major symptom of Parkinson's disease is strong tremor, an involuntary shaking of extremities. Its generation mechanism still is a point of discussion. This is partly due to limitations in acceptable experiments on paticnts and normal human subjects.

On the other hand, part of the uncertainty seems to arise from difficultics in the interpretation of the many experiments performed. Such interpretation requires an insight into the effect of possible pathological changes on tremor generation and on measurable variables. However, the possibly involved parts of the neuromuscular system are so complex that these effects are hard to estimate intuitively.

This can be enhanced by the construction of mathematical models. Such models may at least exclude certain possibilities for tremor generation, and also may indicate which new experiments would discriminate between remaining hypotheses. They might even be helpful in choice, design, and improvement of treatments.

Such models have not been encountered in the selective literature awareness system Parkinson's disease and related disorders (monthly). Therefore this paper illustrates the development and analysis of models of possible Parkinson tremor generation mechanisms. 


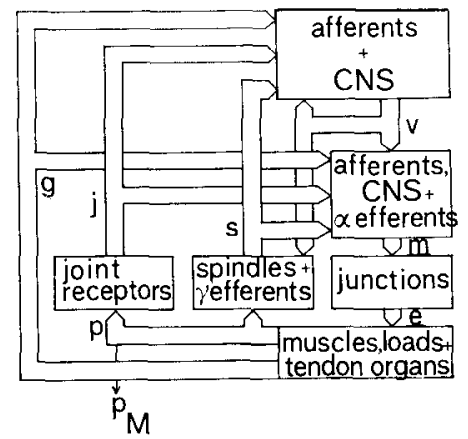

Frg. 1. Signals considered in general tremor generation. These are deviations from the time averages of the following variables: $\mathbf{p}=$ spontaneous position components of extremities, with maximal component $p_{\mathrm{M}}, \mathrm{e}=$ activities in muscle fibers near nerves (single fiber $E M G s$ ), $\mathrm{m}$ $=$ activities in motoneurons near muscles, $\mathrm{s}=$ activities in afferents near spindles, $\mathrm{g}=$ activities in afferents near Golgi tendon organs, $\mathrm{j}=$ activities in afferents near joint receptors, $\mathrm{v}=$ activities in ventrolateral thalamic (VL) cells (CNS $=$ central nervous system).

\section{MODEL VARIABLES}

The model will only concern relations about which information can be obtained from patients. Information is derived from measurements, possibly as an effect of stimulations or artificial changes (e.g., surgery). So the model should only contain variables which can be measured, stimulated, or between which a relation can be changed. Such variables are also relevant in treatment, which involves artificial changes or stimulations, possibly as an effect of measurements.

Consequently, variables which possibly influence tremor have been checked for these properties. This procedure resulted in a model structure which is indicated in Fig. 1. For example, in some patients a part of the ventrolateral thalamus (VL) in the brain is surgically inactivated. Before inactivation, electrical stimulation as well as potential measurements (Holsheimer, 1970) are possible there. Therefore the neuronal activities (action potentials or soma-dendritic potentials) in $\mathrm{VL}$, indicated by vector $\mathrm{v}$, are included into the model variables.

\section{LINEAR MODEL OF PARKINSON-LIKE TREMOR GENERATION}

It will be derived here from the block diagram of Fig. 1, how Parkinson-like tremor might be generated. For this purpose, it is relevant that Parkinson tremors can be considered as narrowband stochastic processes (Ackmann et al., 1977) around a tremor frequency $f_{\mathrm{t}}$ of about $5 \mathrm{~Hz}$.

A narrowband process can be generated as:

-an addition of outputs from a sufficient number of generators with slightly different frequencies,

- the output of a generator with slightly time-varying parameters, or

- the output of a narrowband filter with noise input.

Common to all three are signal loops, of which the open loop response at 


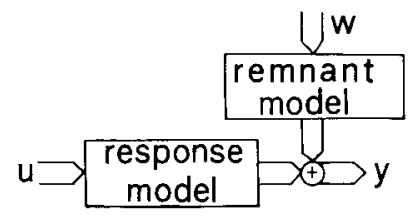

FIG. 2. Linear model of a blcck in Fig. 1. $u=$ inputs, $y=$ outputs, $\mathbf{w}=$ uncorrelated unit spectral density stochastic processes. Response and remnant model contain gains and phases at average amplitudes for frequencies in the input signals.

resonance frequency is unity for the unstable generators and almost unity for the stable filter. Both loop types will be called oscillatory.

If the oscillatory loop is situated completely within one single block in Fig. 1, this block can be represented by Fig. 2. For the filter, the response model contains the oscillatory loop and therefore shows high gain at frequency $f_{\mathrm{t}}$. The remnant model then indicates possible additive noise. For the generator, the remnant model represents the oscillatory loop primarily, with high gain at $f_{t}$. The response model will then have low gain, because variables in the oscillatory loop will often be in the saturating range of nonlinearities.

Oscillatory loops may also involve different blocks in Fig. 1. Such oscillation is expected to occur via one fundamental frequency, and not by successive conversion into super-, resp. subharmonics in successive blocks, because:

- sinusoidal inputs of frequency $f_{\mathrm{t}}$ cause stronger fundamental components than higher harmonics at the outputs of blocks involving $\mathbf{v}$ (Freeman, 1975) and of other blocks (Partridge, 1966), and

- muscles and loads damp higher harmonics of $f_{\mathrm{t}}$ considerably (Partridge, 1966).

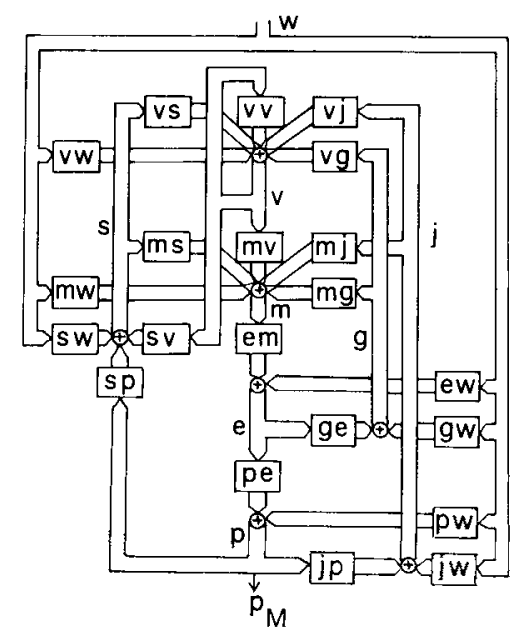

Fig. 3. Frequency response matrices between signals considered. Each block contains the subscripts $y u$ of the frequency response function matrix $\mathbf{H}_{y u}(f)$ from inputs $\mathbf{u}$ to outputs $\mathrm{y}$. The dimension of $\mathrm{w}$ here is higher than in Fig. 2. 


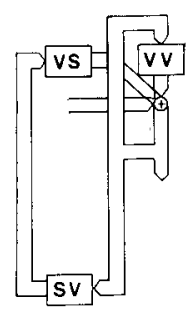

a

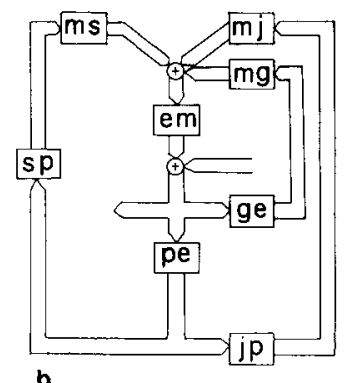

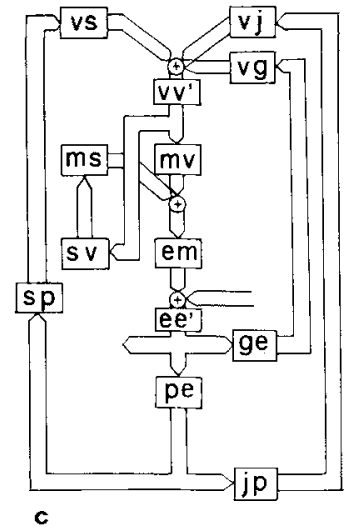

Fig. 4. Closed loop frequency responses. (a) $\mathrm{H}_{v v^{\prime}}$ at $\mathbf{v}$ with loops via VL and spindle nerve fibers closed, (b) $\mathbf{H}_{e e^{\prime}}$ at $\mathbf{e}$ with peripheral loops closed, (c) $\mathbf{H}_{e e^{\prime}}$ at e with all loops closed.

Therefore, also in this case each block may be characterized by Fig. 2. The remnant model then can indicate noise effects.

For easy analysis, each response model is characterized by a frequency response function matrix $\mathbf{H}_{y u}(f)$ for the fundamental frequency $f$ in output vector $\mathbf{y}$ from input vector $\mathbf{u}$. Each remnant model is similarly represented by $\mathbf{H}_{y w}(f)$. Doing so for each block and input vector in Fig. 1 results in Fig. 3.

The effect of each frequency response function in Fig. 3 on $p_{\mathrm{M}}$ is analyzed in Appendix 1. It introduces new equivalent frequency response functions. For stable closed loops these are $\mathbf{H}_{v v^{\prime}}(f)$ from $\mathbf{v}$ to itself with only central loops closed (Fig. 4a), $\mathbf{H}_{e e^{\prime}}(f)$ from e to itself with only peripheral loops closed (Fig. 4b), and $\mathbf{H}_{e e^{\prime \prime}}(f)$ from $\mathbf{e}$ to itself with all loops closed (Fig. 4c). For unstable loops, the

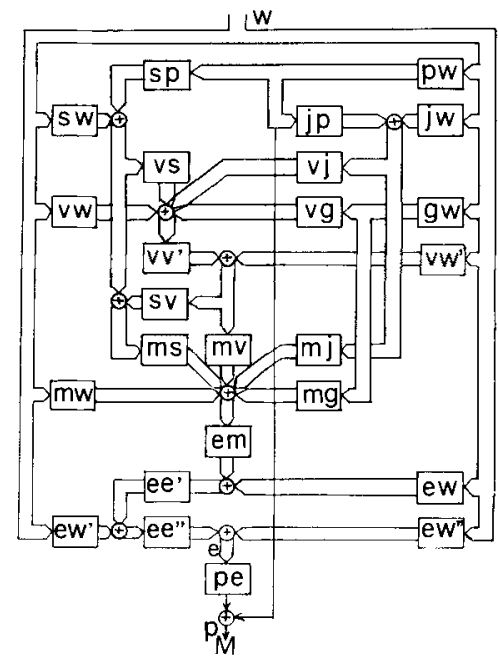

FIG. 5. Loopless linear model of Parkinson-like tremor generation. 


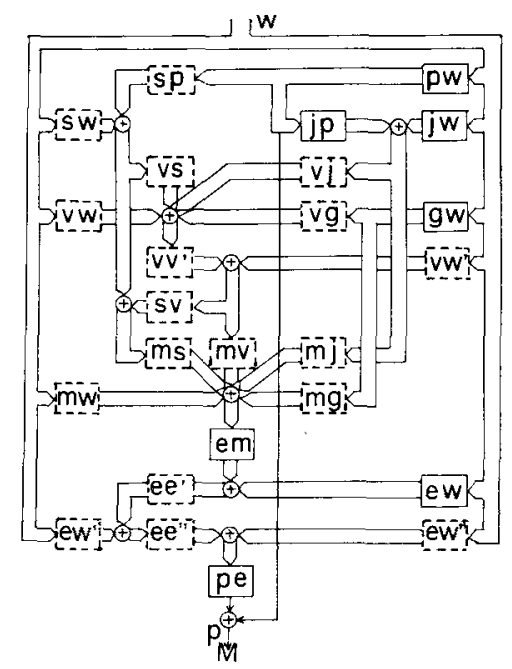

FIG. 6. Hypothetical pathologically increased tremor gains in Parkinson's disease. Solid lines indicate no increase, thick dashed lines hypothetical increases.

generated signals are represented as effects of white noisc filtered by $\mathbf{H}_{v w^{\prime}}(f)$, $\mathbf{H}_{e w^{\prime}}(f)$, and $\mathbf{H}_{e w^{\prime \prime}}(f)$, respectively. With these frequency response functions, the generation of tremor can be represented by Fig. 5 .

In this figure, all signals go from top to bottom without any loops. Therefore, the tremor is the summed effect of the many parallel total frequency response functions $h_{i}(f)$ from clements $w_{i}$ of $\mathbf{w}$ to $p_{\mathrm{M}}$. Because these elements of $\mathbf{w}$ were defined to be uncorrclated, the tromor power or mathematical expectation of $p_{\mathrm{M}}^{2}$ is (Jenkins et al., 1969)

$$
E\left[p_{\mathrm{M}^{2}}\right]=\sum_{i} \int_{0}^{\infty}\left|h_{i}(f)\right|^{2} d f
$$

So the increased tremor power in patients with Parkinson's disease as compared to normal subjects, must be caused by one or more increased $\left|h_{i}(f)\right|$ for $f$ around $f_{\mathrm{t}}$. If each $\mathbf{H}_{y w}(f)$ has different elements of $\mathbf{w}$ as its inputs, similarly to (1) only the gains of elements of $\mathbf{H}_{y u}(f)$ and $\mathbf{H}_{y w}(f)$ for $f \simeq f_{t}$ play a role outside loops. Therefore, only these tremor (frequency) gains, further indicated by $y u$ and $y w$ for shortness, of each block in Fig. 5 will be checked for increase in the next sections.

\section{TREMOR GENERATION HYPOTHESES FROM PARKINSON SUBSTRATE}

In this section, hypothetical increases of tremor gains in Fig. 5 will be inferred from known pathological changes and treatment effects, and indicated in Fig. 6.

It is woll known that in most Parkinson patients certain brain parts are degenerated and show a lack of dopamine, which plays a role in neural transmission. As an effect, these brain parts must be less active. The decrease of tremor by 
drug-induced dopamine increase stresses the relevance of the pathological dopamine shortage. Other Parkinson changes than these are considered late secondary effects (Stark, 1968).

Except by dopamine increase, tremor can be diminished also by surgically inactivating parts of VL (van Manen, 1967). So apparently, in Parkinson's disease the activity in VL is increased, which causes tremor. This increased activity is assumed to result from lower activity in affected parts, which are known to influence VL. Therefore, this influence must be an inhibitory one (Lieberman, 1974).

The increased activity in VL can cause tremor in a number of ways:

Tremor Gains to or from $\mathbf{v}$ (Fig. 3) May be Increased. This could cause tremor for three different generation mechanisms:

- Tremor could be generated elsewhere in the brain and conducted by $\mathbf{v}$. This is equivalent to increased $v w$.

$-v v^{\prime}$ or $v w^{\prime}$ in Fig. 5 might be increased by increase of their constituents $v v, v s$, or $s v$ in Fig. $4 a$.

- The same may be the case with $e e^{\prime \prime}$ or $e w^{\prime \prime}$ in Fig. 5 by their constituents. These are the same as those just mentioned, plus $m v, v j$, or $v g$ in Fig. $4 \mathrm{c}$.

Activity in VL May Influence Parameters. Note that such influences are not represented by signals in the model, but only by parameters in it. Possible parametric influences of VL can be:

-VL may facilitate neural transmission. This can increase all gains montioned above, plus $m w, m g$, and $m j$ in Figs. $4 \mathrm{~b}$ and c. These influence $e e^{\prime}$ or $e w^{\prime}$ and $e e^{\prime \prime}$ or $e w^{\prime \prime}$. Central oscillations may contribute to $s w$ via gamma efferent signals.

-VL may cause constant gamma efferent activities also. These change $\mathbf{H}_{\varepsilon p}(f)$ and therefore may increase gains of blocks which contain it, like $e e^{\prime}$ or $e w^{\prime}$ and $e e^{\prime \prime}$ or $e w^{\prime \prime}$.

In the brain, tremor may be generated in many different ways (Andersen et al., 1968). On the other hand, quantitative data are available about the spindle loop (left loop in Fig. 4b). So the possibility of that loop to oscillate is relatively easily checked. This is performed in Appendix 2. The result shows that these elements $e e^{\prime}$ or $e w^{\prime}$ might very well be increased. These increases would result from increased static sensitivities $s_{\mathrm{s}}$ of spindles more than from dynamic ones $s_{\mathrm{d}}$ in $\mathbf{H}_{s p}(f)$. Effects from increased $m s$ are intermediate.

The foregoing would exclude increased $p e, p w, e m, e w, j p, j w$, and $g w$. This has bcen indicated in Fig. 6 .

\section{POSSIBLE PARKINSON TREMOR GENERATION MECHANISMS FROM LITERATURE ABOUT EXPERIMENTS ON PATIENTS}

The former section shows several hypotheses for Parkinson tremor generation. Many of these changes have been induced artificially in animals. Generally, they indeed elicited Parkinson-like tremor (e.g., Gybels, 1963). However, the mecha- 


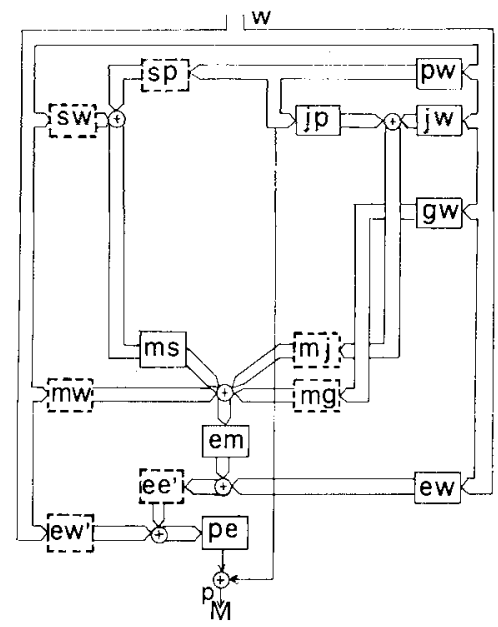

Fig. 7. Possibly increased tremor gains in some Parkinson patients. Thick dashed lines indicate these gains.

nisms of these may differ from the one(s) in Parkinson patients. So actual Parkinson tremor generation mechanisms may be investigated only by experiments on patients.

Therefore, known litcrature about expcriments on Parkinson patients has bcen checked for explicit results concerning increased or normal tremor gains of dashed blocks in Fig. 6. The results will be summarized below and indicated in Fig. 7.

After a tap on the tendon (Gybels, 1963) or nerve stimulation (Alberts et al., 1965), the next top in e occurs one tremor period afterwards. This is independent of the original tremor phase, which generally is resct. Tremor phase is reset also by sudden shortening of a muscle, caused by removal of a load. This involves temporary inactivation of spindles (Angel et al., 1969). Such resetting is possible only, if these stimuli influence a tremor generating loop in saturation. Both responses are so quick that only the peripheral loops, shown in Fig. 4b, can be involved. So $e e^{\prime}$ or $e w^{\prime}$ must be increased.

After stimulation of nerves, in e first an efferent response via $\mathbf{H}_{e m}(f)$ is seen, followed by an afferent one via $\mathbf{H}_{e m}(f) \mathbf{H}_{m s}(f)$. This second response is reported to have a normal (e.g., Dietrichson, 1973; Sica et al., 1972) as well as $1 \frac{1}{2}$ times as high (McLellan, 1973) ratio to the first one. This implies normal or slightly increased $m s$. Finally, a very late response via the brain occurs. In patients with Parkinson tremor, this response is similar to the normal one (Tatton et al., 1975). So the contribution to $m s$ via the brain appears normal too.

Measurements with microelectrodes in peripheral nerves dominantly contain effects of s. Their response to movement is within a few milliseconds, and therefore via peripheral loops. The component proportional to position is increased and that to velocity normal (Hagbarth et al., 1970). This indicates constant $s_{\mathrm{d}}$ with increased $s_{\mathrm{s}}$ and therefore increased $e e^{\prime}$ or $e w^{\prime}$.

Responses of e to $\mathbf{p}$ have been described, e.g., by Lance et al. (1963), Andrews 
et al. (1972), and Dietrichson (1973). Dynamic responses were about normal, which together with normal $m s$ indicates normal $s_{\mathrm{d}}$. Static responses were higher than normal, indicating increased $s_{\mathrm{s}}$ and therefore $s p$ and $e e^{\prime}$. They were diminished by temporary probable inactivation of $s$ by local anesthesia around a nerve, intramuscular injection of procaine, or interruption of blood supply by a pressure cuff. Parkinson tremor then also decreased (Lance et al., 1963) as well as continued in one patient (Walshe, 1924). Inactivation of s certainly was achieved by section of dorsal roots from an arm, which replaced Parkinson tremor by another type (Pollock and Davis, 1930). This may indicate normal $s_{\mathrm{s}}$ or pathologically increased $s_{\mathrm{s}}$ with inhibition of another oscillatory loop by $\mathbf{s}$.

Strong electrical stimulation of VL causes movement. This is used for surgery localization. This only stimulates other brain parts than VL however, as local stimulation by sufficiently low intensities does not influence tremor linearly. Instead, it generally augments (Gybels, 1963; Alberts et al., 1965) or depresses (Kandel et al., 1974) tremor amplitude and frequency (Lücking, 1976). Moreover, tremor frequencies are present in VL while not in $\mathbf{p}$ (Lücking, 1976). So both $m v$ and $s v$ are zero. This eliminates the fecdback in Figs. $4 \mathrm{a}$ and $c$, so $e e^{\prime \prime}$ and $v v^{\prime}$ become unity and therefore can be omitted. Yet correlation has been found between $\mathbf{p}$ and microelectrode measurements of $\mathbf{v}$ for some cells (Andrew et al., 1972). This must have arisen from afferent effects of $\mathbf{p}$ on $\mathbf{v}$ (Andrew et al., 1972) or from artifacts in $\mathbf{v}$ from head tremor, corrclated with other tremors.

Figure 7 shows the resulting models of possible tremor generation mechanisms for the paticnts concerned.

\section{CONCLUSIONS}

Figure 5 shows how Parkinson-like tremors may be generated. Figure 6 shows hypotheses for Parkinson tremor generation according to known discase substrate. For the patients about which explicitly interpretable experiments were found in the literature, Fig. 7 represents the possibly increased gains which may cause their tremor. This shows that for some of these patients tremor could be generated in peripheral reflex loops, for some possibly in other loops. These crucial experiments might be repeated for more patients. They might indicate different oscillatory loops for different patients.

Figures 6 and 7 may be helpful for the design of new experiments, which might explicitly differentiate between remaining possibilities.

Effective treatment will have to interfere with the highest gains from $\mathrm{w}$ to $p_{M}$ in Fig. 7, preferably but not necessarily, in the possibly increased trcmor gains, if possible only in the pathologically changed frequency responses. Design of such a treatment may be served by constructing a specific version of the model per treatment type. Parameters of these should then be estimated per individual patient.

\section{LIST OF SYMBOLS}

This list only contains symbols in the main text. Symbols in appendices may deviate and are defined there explicitly. Explanations below are short and 
primarily moant for recognition; for exact definitions the reader is referred to the text.

\begin{tabular}{|c|c|c|c|}
\hline$e$ & $=$ electrical activity in muscle & $s_{\mathrm{d}}$ & sensitivity \\
\hline$f$ & $=\mathrm{fr}$ & $s_{\mathrm{s}}$ & $\begin{array}{l}\text { itivity } \\
\text { a block }\end{array}$ \\
\hline$f_{\mathrm{t}}$ & $=$ frequency of tremor $\simeq 5 \mathrm{~Hz}$ & & Fig. 1 \\
\hline g & $\begin{aligned}= & \text { Golgi tendon organ afferent } \\
& \text { activity vector }\end{aligned}$ & $\begin{array}{l}\mathbf{v} \\
\mathrm{VL}\end{array}$ & $\begin{array}{l}=\mathrm{VL} \text { activity vector } \\
=\text { ventrolateral thalamus }\end{array}$ \\
\hline $\mathbf{H}_{y u}(f)$ & $\begin{array}{l}\text { response function } \\
\mathrm{y} \text { from } \mathfrak{u}\end{array}$ & $\mathrm{w}$ & $\begin{array}{l}=\text { white uncorrelated noise } \\
\text { vector }\end{array}$ \\
\hline$h_{i}(f)$ & $\begin{array}{l}\text { icy response function } \\
\text { i to } p_{\mathrm{M}}\end{array}$ & $w_{i}$ & $\begin{array}{l}=\text { element } i \text { of } \mathrm{w} \\
=\text { output vector of a block in }\end{array}$ \\
\hline & $=$ joint receptor afferent activ- & & Fig. 1 \\
\hline $\mathbf{m}$ & $=$ motoneuron activity vector & $y u$ & $\begin{array}{l}=\text { tremor gain }=\text { element } \\
\left|\mathbf{H}_{y u}\left(f_{\mathrm{t}}\right)\right|\end{array}$ \\
\hline p & $=$ position of extremities vector & $y u^{\prime}$ & $=$ tremor gain for single closed \\
\hline & al comp & & loop \\
\hline & $\begin{array}{l}=\text { spindle afferent activity } \\
\text { vector }\end{array}$ & $y u^{\prime \prime}$ & $\begin{aligned}= & \text { tremor gain for double closed } \\
& \text { loop. }\end{aligned}$ \\
\hline
\end{tabular}

APPENDIX 1. SOLUTION OF $p_{M}$

For inputs $\mathbf{u}(t)$ and outputs $\mathbf{y}(t)$ of cach block in Fig. 3, sample Fourier transforms are defined as

$$
\mathbf{u}_{T}(f)=\frac{1}{T} \int_{-T^{\prime} / 2}^{+T / 2} \mathbf{u}(t) e^{-j 2 \pi s t} d t
$$

and $\mathrm{y}_{T}(f)$ similarly. If $1 / T$ is taken small compared to the frequency difference over which $\mathbf{H}_{y u}(f)$ varies considerably (Jenkins et al., 1969),

$$
\mathrm{y}_{T}(f) \simeq \mathbf{H}_{y u}(f) \mathbf{u}_{T}(f) .
$$

For notational simplicity, (3) will be indicated as

$$
y=H_{y u} u \text {. }
$$

Figure 3 shows that $\mathbf{v}$ and $\mathbf{e}$ are central and peripheral signals on which all other signals depend. Therefore, these will be solved. As $p_{\mathrm{M}}$ most directly depends on $\mathbf{e}, \mathbf{v}$ is solved first as a function of $\mathbf{e}$.

From Fig. 3 it can be seen that

$$
\begin{aligned}
& v=H_{v v} v+H_{v s} s+H_{v j} j+H_{v g} g+H_{v w} w \\
& s=H_{s v} v+H_{s p}\left(H_{p e} e+H_{p w} w\right)+H_{s w} w \\
& j=H_{j p}\left(H_{p e} e+H_{p w} w\right)+H_{j w} w \\
& g=H_{g e} e+H_{g w} w
\end{aligned}
$$


Substitution of (6)-(8) into (5) and solution of $v$ yields

$$
\begin{gathered}
v=H_{v v^{\prime}}\left(\left(H_{v s} H_{s p}+H_{v j} H_{j p}\right)\left(H_{p e} e+H_{p w} w\right)+H_{v s} H_{s w} w\right. \\
\left.\quad+H_{v j} H_{j w} w+H_{v g}\left(H_{y e} e+H_{g w} w\right)+H_{v w} w\right)+H_{v w} w \\
H_{v v^{\prime}}=\left(I-H_{v v}-H_{v s} H_{s v}\right)^{-1}
\end{gathered}
$$

with $I$ the identity matrix. The composition of $H_{v v^{\prime}}$ is indicated in Fig. 4a. $H_{v w^{\prime}}$ in (9) represents signals generated in the loops indicated by $H_{v v^{\prime}}$.

Figure 3 also shows

$$
e=H_{e m}\left(H_{m v} v+H_{m s} s+H_{m j} j+H_{m_{\theta}} g+H_{m w} w\right)+H_{e w} w .
$$

Substitution of (6)-(8) into (11) and solution of $e$ yields

$$
\begin{gathered}
e=H_{e e^{\prime}}\left(H _ { e m } \left(H_{m v} v+H_{m s}\left(H_{s v} v+H_{s p} H_{p w} w+H_{s w} w\right)\right.\right. \\
+H_{m j}\left(H_{j p} H_{p w} w+H_{j w} w\right) \\
\left.\left.+H_{m g} H_{g w} w+H_{m w} w\right)+H_{e w} w\right)+H_{e w} w \\
H_{e e^{\prime}}=\left(I-H_{e m}\left(\left(H_{m s} H_{e p}+H_{m j} H_{j p}\right) H_{p e}+H_{m g} H_{g e}\right)\right)^{-1}
\end{gathered}
$$

indicated in Fig. $4 \mathrm{~b} . H_{e w^{\prime}}$ in (12) represents signals generated in the loops indicated by $H_{e e^{\prime}}$.

Substitution of (9) into (12) and solution of $e$ yields

$$
\begin{aligned}
e= & H_{e e^{\prime \prime}}\left(H _ { e e ^ { \prime } } \left(H _ { e m } \left(\left(H_{m v}+H_{m s} H_{s v}\right)\right.\right.\right. \\
& \times\left(H_{v v^{\prime}}\left(H_{v s}\left(H_{s p} H_{p w}+H_{s w}\right)+H_{v j}\left(H_{j p} H_{p w}+H_{j w}\right)+H_{v g} H_{o w}\right)+H_{v w^{\prime}}\right) \\
& \left.+H_{m s}\left(H_{s p} H_{p w}+H_{s w}\right)+H_{m j}\left(H_{j p} H_{p w}+H_{j w}\right)+H_{m w}\right) \\
& \left.\left.+H_{e w}\right)+H_{e w^{\prime}}\right) w+H_{e w^{\prime}} w, \quad \text { (14) } \\
H_{e e^{\prime \prime}}= & \left(I-H_{e e^{\prime}} H_{e m}\left(H_{m v}+H_{m s} H_{s v}\right) H_{v v^{\prime}}\right. \\
& \left.\quad \times\left(\left(H_{v s} H_{s p}+H_{v j} H_{j p}\right) H_{p e}+H_{v g} H_{g e}\right)\right)^{-1}
\end{aligned}
$$

indicated in Fig. 4c. $H_{e w^{\prime \prime}}$ in (14) represents signals generated in the loops indicated by $H_{e e^{\prime \prime}}$. Equation (14) and vector $\mathbf{h}_{p e}(f)$ from e to $p_{\mathrm{M}}$ can be represented in the form of Fig. 5, which contains each $\mathrm{H}$ only once.

\section{APPENDIX 2. HYPOTHETICAL TREMOR GENERATION BY PERIPHERAL SPINDLE LOOP}

In this appendix, effects of changes in $\mathbf{H}_{m s}(f)$ and $\mathbf{H}_{s p}(f)$ on $e e^{\prime}$ will be investigated. For use of experimental data from the literature, some simplifying definitions are needed:

$-p=$ displacement of lower arm at the insertion of upper arm muscles, with fixed upper arm,

$-e=$ difference between the average of all elements of $\mathrm{e}$ in biceps, and the same in triceps, and

$-s, m=$ the same as $e$ for $\mathbf{s}$ and $\mathbf{m}$, respectively.

As an effect of these definitions, reciprocal inhibition is accounted for by scalar 
representation $h_{m s}(f)$ of $\mathbf{H}_{m s}(f)$. For maximal accuracy, data from the largest investigated loop components will be used.

The time from afferent stimulation to efferent response in a human arm is 0.02 sec. (Magladery, 1955). This yiclds

$$
h_{m s}(f)=A e^{-j \delta / 8}
$$

with unknown $A$ and with $m$ and $s$ expressed in pulscs per second.

Open loop experiments on other blocks in the loop can be performed for animals only. Thus $s$ from sinusoidal length changes of cat muscle (Matthews et al., 1969) can be approximated by

$$
h_{s p}(f)=s_{\mathrm{s}}\left(1+\frac{s_{\mathrm{d}}}{s_{\mathrm{s}}} j 2 \pi f\right)
$$

with $p$ expressed in millimeters and with $s_{\mathrm{s}}$ the static and $s_{\mathrm{d}}$ the dynamic spindle sensitivity, with $s_{\mathrm{s}}=7$ to 95 and $s_{\mathrm{d}} / s_{\mathrm{s}}=0.08$ normally.

The effect of sinusoidal modulations of the motoneurons stimulation pulse rate on cat muscle length for any physiological inertial load plus gravitation (Partridge, 1966) can be approximated by

$$
h_{p e}(f) h_{e m}(f)=\frac{-0.25}{(1+j f / 0.6)(1+j f / 1.8)(1+j f / 3.6)} .
$$

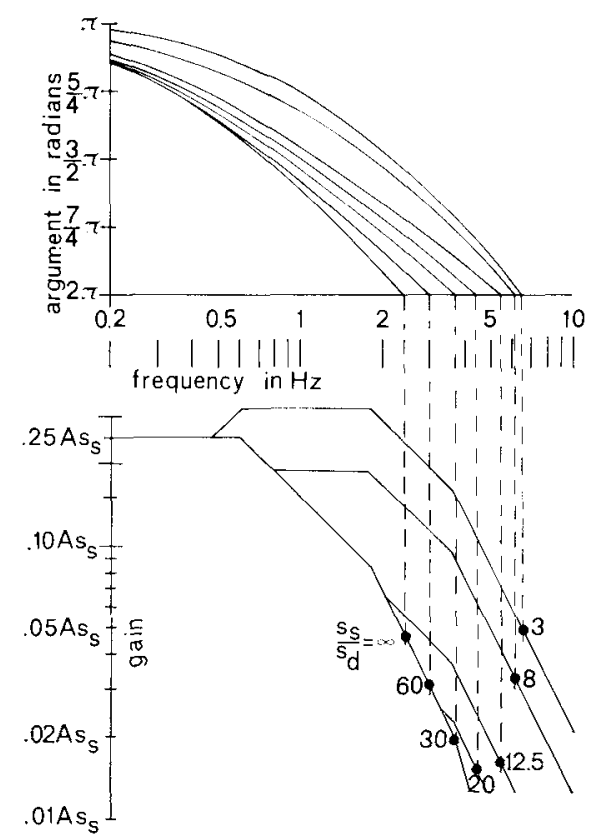

FIG. 8. Phase and gain asymptote plots of open peripheral spindle loop for several ratios between static and dynamic spindle sensitivity. The value of a specific ratio $s_{\mathrm{s}} / s_{\mathrm{d}}$ is shown near the dot on the specific gain asymptote at the frequency for which the specific phase is $2 \pi$ radians. 
The spindle loop would oscillate, if the open loop frequency response function from $(16)-(18)$

$$
\begin{aligned}
& h_{p e}(f) h_{e m}(f) h_{m s}(f) h_{s p}(f) \\
& =-0.25 A s_{\mathrm{s}} \frac{e^{-j / / 8}\left(1+\frac{s_{\mathrm{d}}}{s_{\mathrm{s}}} j 2 \pi f\right)}{(1+j f / 0.6)(1+j f / 1.8)(1+j f / 3.6)} \simeq 1 .
\end{aligned}
$$

(19) is shown in Fig. 8. The dots there indicate the approximate gains for which the phase is $2 \pi$ radians, and therefore unstability at the related frequency would occur if this gain would be unity.

It appears that for a certain $s_{\mathrm{s}}$ and $A$, the normal $s_{\mathrm{s}} / s_{\mathrm{d}}=12.5$ is near the value for which most extra gain is needed for oscillation. Tremor generation can then arise from two types of changes:

$-s_{\mathrm{s}} / s_{\mathrm{d}}$ remains the same, but the gain factor $A s_{\mathrm{s}}$ increases. This can be caused by increased $A$ or by proportional increase of $s_{\mathrm{s}}$ and $s_{\mathrm{d}}$ together. Then for sufficient $A s_{\mathrm{s}}$, the loop would oscillate near $5 \mathrm{~Hz}$. This happens to be the average $f_{\mathrm{t}}$.

- Fither $s_{\mathrm{s}}$ or $s_{\mathrm{d}}$ is increased, so that the gain at $2 \pi$ radians phase shift becomes higher than the minimum in Fig. 8.

If only $s_{\mathrm{s}}$ would be increased, $A s_{\mathrm{s}}$ as well as $s_{\mathrm{s}} / s_{\mathrm{d}}$ would increase. Therefore, then both mentioned effects would combine to give a strong gain increase at $2 \pi$ radians phase shift.

If $s_{\mathrm{s}} / s_{\mathrm{d}}$ increases, stronger tremor is accompanied by lower tremor frcquency. This also occurs in the transition (Stiles et al., 1976) of weak $9-\mathrm{Hz}$ normal-like Parkinson hand tremor to strong tremor. If normal tremor would be generated in the spindle loop, the minimum in Fig. 8 would be expected at $9 \mathrm{~Hz}$, however. The difference between that frequency and the $5 \mathrm{~Hz}$ of the model could be caused by differences between human parameters and those of cats. The range of $f_{\mathrm{t}}$ from 3 to $8 \mathrm{~Hz}$ for different patients may be accounted for by individual parameter differences, mainly those of $s_{\mathrm{s}}$ and $s_{\mathrm{d}}$.

Stein et al. (1976) expected the minimum to occur at lower $s_{\mathrm{d}} / s_{\mathrm{s}}$ than normal. The difference may partly be caused by the use of data from complete subsystem experiments in this appendix.

\section{ACKNOWLEDGMENTS}

The author wants to express his admiration and gratitude to the late Dr. G. J. van Hoytema. His profound interest in this subject was an indispensable inspiration to work on it.

Further he likes to thank staff and students of the Bio-information Group of Twente University of Technology, Dr. J. van Manen and the members of the Movement Control Group of the Foundation of Biophysics in the Netherlands for discussions concerning Parkinson's disease, Prof. E. W. Gröneveld and Prof. H. Kwakernaak for methodological advice, and Miss G. G. M. Steijlen for drawings and typework.

\section{REFERENCES}

Ackmann, J. J., Sances, A., Jr., Larson, S. J., and Baker, J. B. Quantitative evaluation of longterm Parkinson tremor. IEEE Transactions on Biomedical Engineering 1977, BME-24, 49-56.

Alberts, W. W., Libet, B., and Wright, E. W., Jr. Physiological mechanism of tremor and rigidity in Parkinsonism. Confinia Neurologica 1965, 26, 318-327. 
Andersen, P., and Andersson, S. A. Models of rhythmic activity. Physiological basis of the alpha rhythm, New York: Appleton-Century-Crofts, 1968. Chap. 16.

Andrews, C. J., Burke, D., and Lance, J. W. The response to muscle stretch and shortening in Parkinsonian rigidity. Brain 1972, 95, 795-812.

Andrew, J., and Rudolf, N. de M. Micro-electrode recordings in the human thalamus during stereotaxic surgery. In J. P. Nicholson (Ed.), Interdisciplinary investigation of the brain. London: Plenum, 1972, Pp. 53-72.

Angel, R. W., Aguilar, J. A., and Hofmann, W. W. Action tremor and thalamotomy. Electroencephalography and Clinical Neurophysiology 1969, 26, 80-85.

Dietrichson, P. The fusimotor system in relation to spasticity and Parkinsonian rigidity. Scandinavian Journal of Rehabilitation Medicine 1973, 5, 174-178.

Freeman, W. J. Mass action in the nervous system. New York: Academic Press, 1975.

Gybels, J. M. The neural mechanism of Parkinson tremor. Brussels: Arscia S. A., 1963.

Hagbarth, K.-E., Hongell, A., and Wallin, G. Parkinson's disease: Afferent muscle nerve activity in rigid patients. Acta Societas Medicorum Upsaliensis 1970, 74, 70-76.

Holsheimer, J. Physiological records from the ventrolateral thalamus during stereotactic operations on Parkinsonian patients. Report from a Multidisciplinary Team. Psychiatria, Neurologia, Neurochirurgia 1970, 73, 339-352.

Jenkins, G. M., and Watts, D. G. Spectral analysis and its applicalions. San Francisco: HoldenDay, 1968.

Kandel, E. I., Aizerman, M. A., Andreeva, E. A., Tenenbaum, L. A., and Shafranova, E. I. Interrelations of resting, postural and action tremor in Parkinsonian patients before and after surgery. Confinia Neurologica 1974, 36, 356-359.

Lance, J. W., Schwab, R. S., and Peterson, F. A. Action tremor and the cogwheel phenomenon in Parkinson's disease. Brain 1963, 86, 95-110.

Lieberman, A. N. Parkinson's disease: A clinical review. The American Journal of the Medical Sciences 1974, 267, 66-90.

Lücking, C. H. Stereotaktische Behandlung des Tremors. Stuttgart: Thieme Verlag, 1976.

Magladery, J. W. Some observations on spinal reflexes in man. Pfuegers Archiv 1955, 261, 302-312.

Matthews, P. B. C., and Stein, R. B. The sensitivity of muscle spindle afferents to small sinusoidal changes of length. Journal of Physiology 1969, 200, 723-743.

McLellan, D. L. Dynamic spindle reflexes and the rigidity of Parkinsonism. Journal of Neurology, Neurosurgery, and Psychiatry 1973, 36, 342-349.

Parkinson's disease and related disorders, citations from the literature. U.S. Department of Health, Monthly.

Partridge, L. D. Signal-handling characteristics of load-moving skeletal muscle. American Journal of Physiology 1966, 210, 1178-1191.

Pollock, L. J., and Davis, L. Muscle tone in Parkinsonian states. Archives of Neurology and Psychiatry 1930, 23, 303-319.

Sica, R. E., Aguilera, N., and Herskovits, E. Impaired potentiation of $H$-reflexes in patients with Parkinson's disease. Medicina 1972, 32, 599-595.

Stark, L. Neurological control systems. Studies in bioengineering. New York: Plenum Press, 1968. Pp. 338-347.

Stein, R. B., and Oguztöreli, M. N. Does the velocity sensitivity of muscle spindle stabilize the stretch reflex? Biological Cybernetics 1976, 23, 219-228.

Stiles, R. N., and Pozas, R. S. A mechanical-reflex oseillator hypothesis for Parkinsonian hand tremor. Journal of Applied Physiology 1976, 40, 990-998.

Tatton, W. G., and Lee, R. G. Evidence of abnormal long-loop reflexes in rigid Parkinsonian patients. Brain Research 1975, 100, 671-676.

van Manen, J. Stereotactic methods and their applications in disorders of the motor system. Assen: Van Gorcum, 1967.

Walshe, F. M. R. Observations on the nature of the muscular rigidity of paralysis agitans, and on its relationship to tremor. Brain 1924, 47, 159-177. 